\title{
Evaluation of Activities of Daily Living/ Instrumental Activities of Daily Living to Accurately Determine Severity of Moderate and Severe Alzheimer's Disease: Comparison of Assessments by Receiver Operating Characteristic Curve and Discriminant Analyses
}

\author{
Yukiko Suzukia Koji Teruyab Hideki Mochizukia ${ }^{\mathrm{b}} \quad$ Akira Nagasawa $^{\mathrm{c}}$ \\ Tomoko Kondo a Nobuaki Shimodad

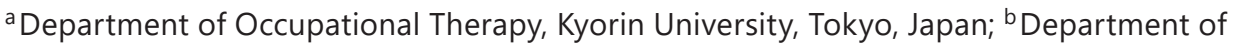 \\ Public Health, Faculty of Health Sciences, Kyorin University, Tokyo, Japan; ' Department of \\ Rehabilitation, Juntendo Tokyo Koto Geriatric Medical Center, Tokyo, Japan; dDepartment of \\ Rehabilitation, Faculty of Health Sciences, Tokyo Kasei University, Sayama, Japan
}

Keywords

Activities of daily living · Alzheimer's disease · Discriminant analyses · Evaluation

\begin{abstract}
Aim: The aim of this study was to determine Activities of Daily Living/Instrumental Activities of Daily Living (ADL/IADL) evaluations that will enable better understanding of the severity of Alzheimer's disease (AD). Methods: AD patients were evaluated by Functional Independence Measure (FIM), Hyogo Activities of Daily Living Scale (HADLS), and Assessment of Motor and Process Skills (AMPS) to identify the assessments that will enable highly precise discrimination of AD Clinical Dementia Rating (CDR) 2 (moderate) and CDR3 (severe) using receiver operating characteristic (ROC) curve and discriminant analyses. Results: The participants were 75 AD patients $(C D R 2=50$, mean age $=80.3 \pm 5.9$ years; $C D R 3=25$, mean age $=78.3 \pm 9.0$ years). The evaluation methods consisted of FIM, HADLS, and AMPS. The results were divided into FIM-M, FIM-C, HADLS-ADL, HADLS-IADL, AMPS-motor skills, and AMPS-process skills. The values for the area under the curve (AUC) were compared by ROC curve and discriminant analyses. AUC values for FIM-C and AMPS-process skills were 0.956 and 0.947 , respectively. With these two evaluations only, values $\geq 0.9$ were shown. Moreover, the AUC of the discrimination score (combination of the FIM-C and AMPS-process skills) was significantly higher than those for FIM-M, FIM-C, HADLS-ADL, HADLS-IADL, and AMPS-motor skills. Conclusions:
\end{abstract}


The results demonstrated that evaluation by FIM-C and AMPS-process skills methods was useful for discriminating between CDR2 (moderate) and CDR3 (severe) AD. Moreover, the results indicated that these two evaluation methods enabled more accurate determination of severity and the spared capabilities of AD patients.

(C) 2019 The Author(s)

Published by S. Karger AG, Basel

\section{Introduction}

For effective occupational therapy of Alzheimer's disease (AD) patients, abilities to perform Activities of Daily Living (ADL), Instrumental Activities of Daily Living (IADL), and work performance need to be evaluated and the resulting information directly connected to an occupational therapy plan. Because patients with moderate and severe AD are assisted through many ADL and IADL programs, ADL and IADL evaluations that enable proper discrimination of the severity of AD are important.

Clinical Dementia Rating (CDR) is an evaluation method commonly used worldwide [1]. Beyond using CDR to obtain information linked to an AD occupational therapy program, ADL/ IADL performance and work performing skills of AD patients need to be directly evaluated.

In CDR, the "home and hobbies" section best shows the difference between moderate and severe AD [1]. A CDR2 (moderate) rating of this section indicates AD patients who can perform simple household chores, whereas a CDR3 (severe) rating indicates those who can hardly perform household chores [1, 2]. However, among AD patients who require assistance with $\mathrm{ADL}$, such as dressing and toileting, some can perform some IADL. For example, we have worked with many severe AD patients who found it difficult to understand movement directives and required guidance for all ADL but could slowly fold a towel or apron and place it properly without guidance. Although a lack of memory regarding when, where, and what is common in AD patients [3], their procedural memory is often relatively spared [4]. Even severe CDR3 patients are known to retain the ability to perform activities they were engaged in for many years as lifestyle habits. Understanding this reality is important for an occupational therapist so that this spared capability is not overlooked.

Methods used to evaluate ADL and IADL performance include Functional Independence Measure (FIM) [5], Hyogo Activities of Daily Living Scale (HADLS) [6], and Assessment of Motor and Process Skills (AMPS) [7, 8]. However, these methods have various advantages and disadvantages when evaluating patients with AD. With FIM, the level of self-reliance for individual ADL items (such as eating and grooming) can be determined quantitatively, based on the amount of assistance required. Moreover, it can be used to evaluate cognitive functions, including communication ability. However, FIM is not effective in evaluating a patient's ability to perform IADL, even for severe $\mathrm{AD}$ patients, or the range of skills that enable independent performance of ADL. HADLS was developed as an evaluation method specifically for AD patients, and when used with ADL, even IADL can be evaluated. It is also clinically effective in severe AD patients [9]. However, as this system is usually performed via an interview, providers of reliable information are required, which can be a severe disadvantage in the case of AD patients. Fortunately, IADL that cannot be addressed by FIM can be evaluated with AMPS, wherein common ADL and IADL problems in daily living can be selected and evaluated; therefore, the spared skills of the patients can be evaluated in detail by procedural memory. Unfortunately, AMPS cannot evaluate communication performance or the level of independence and the amount of assistance required for various ADL and IADL. As such, we aimed to clarify which method or combination of methods using FIM, HADLS, and AMPS would best enable the discrimination between CDR2 (moderate) and CDR3 (severe) AD. 
Table 1. Participants' characteristics and evaluation results

$n$ (CDR2/CDR3)

Men/women, $n$

Age, years (range)

Years of education after graduation from elementary school (range)

Complications including overlapping, $n$

Cerebrovascular diseases

Diseases of internal medicine

Diseases of orthopedics

Parkinson's syndrome

MMSE, score

FIM-Total, score

FIM-M, score

FIM-C, score

HADLS-Total, score

HADLS-ADL, score

HADLS-IADL, score

AMPS-motor skills, logits

AMPS-process skills, logits
$75(50 / 25)$

$38 / 37$

$79.6 \pm 7.1(60-96)$

$5.7 \pm 3.2(0-11)$

57

60

21

$10.1 \pm 5.9$

$81.1+21.3$

$64.1 \pm 17.6$

$17.0 \pm 5.3$

$54.91 \pm 19.77$

$23.09 \pm 15.09$

$31.83 \pm 6.27$

$1.64 \pm 0.68$

$0.30 \pm 0.72$

To determine whether the Five Cognitive Tests (memory, attention, language, visuospatial function, and reasoning) can be applied for screening mild dementia, Sugiyama et al. [10] evaluated CDR0 (none) and CDR0.5 (questionable) elderly patients using the Five Cognitive Tests with the Mini-Mental State Examination (MMSE). They also calculated the area under the curve (AUC), cutoff values, sensitivity, and specificity using a receiver operating characteristic (ROC) curve analysis. The results indicated that, compared with MMSE, CDR0 (none) and CDR0.5 (questionable) groups could be discriminated with high precision using the Five Cognitive Tests.

Based on these previous findings, we used the method by Sugiyama et al. [10] as reference and aimed to determine evaluations or evaluation combinations of FIM, HADLS, and AMPS that will enable precise discrimination between CDR2 and CDR3 AD using ROC curve and discriminant analyses. A successful evaluation should measure the severity of CDR2 and CDR3 AD and the differences in the spared capabilities of the patients. To proceed with this mission, we categorized the evaluation methods into FIM-motor skills (FIM-M), FIM-cognitive skills (FIM-C), HADLS-ADL, HADLS-IADL, AMPS-motor skills, and AMPS-process skills. Finding the most effective combination of these methods would better enable occupational therapists to evaluate ADL and IADL in detail and therefore better design highly productive occupational therapy programs.

\section{Materials and Methods}

\section{Participants}

The participants were $75 \mathrm{AD}$ patients currently hospitalized in a dementia ward. The participants were patients diagnosed with $\mathrm{AD}$ by a physician based on the tenth edition of the International Classification of Diseases (ICD-10). Of the participants, 50 were CDR2 (moderate) and 25 were CDR3 (severe). Patients who could not follow verbal instructions, with clearly severe physical illness or unstable chronic disease, and with serious visual disorders were excluded (Table 1). 
This study was approved by the ethics committee of Juntendo Tokyo Koto Geriatric Medical Center (approval number 75-1) and the ethical review board of the Faculty of Health Sciences, Kyorin University (approval number 26-49). Written informed consent was obtained from all subjects or their families for publishing the data used in this study.

\section{Evaluation}

The evaluation items consisted of CDR [2], FIM [5], HADLS [6], and AMPS [7, 8].

CDR [2] was assessed by evaluating memory, orientation, judgment and problem solving, community affairs, home and hobbies, and personal care, each on a 5-point scale and based on the clinical information and behavioral observation of the patients. CDR consisted of a simple set of criteria for comprehensive evaluation of severity of dementia. The levels of severity were CDR0 (none), CDR0.5 (questionable), CDR1 (mild), CDR2 (moderate), and CDR3 (severe).

The evaluation items in FIM [5] consisted of two major items, namely, FIM-M (13 items) and FIM-C (5 items). Depending on the level of independence and level of assistance needed, each item was scored on a 7-point scale. The lower the score was, the larger was the amount of assistance required, thus indicating a low level of independence. Maximum and minimum scores in FIM-M were 91 and 13 points, respectively, while those in FIM-C were 35 and 5 points, respectively. The maximum and minimum scores for FIM-Total were 126 and 18 points, respectively.

HADLS [6] is the overall impairment scale for ADL and IADL in AD patients. In the evaluation based on caretaker interview, 18 of the items on daily living activities were evaluated on a 3- to 7-point scale. In addition, of the lower-ranking items of HADLS, the total score for the 8 items on ADL (toileting, eating, dressing, grooming, wash, tooth brushing/wash dentures, bathing, and locomotion) was set as "HADLS-ADL," and that for the 10 items on IADL (using a telephone, shopping, cooking, cleaning up, bed making, cleaning up after eating, laundry, using fire, using switches, and financial management) was set as "HADLS-IADL"; both were used as objects of the analysis.

AMPS $[7,8]$ measures the minimum units of behavior, namely, motor skills and process skills, and is said to evaluate the qualitative aspects of ADL. Motor skills are required to perform specific body movements in order to accomplish a task. Process skills are required to adapt one's actions according to the situation when performing a task. In the evaluation, participants select two tasks that match their interest and ability from a list of 125 tasks standardized by their level of difficulty. The participants are then observed as they perform those tasks. The 16 items on motor skills and 20 items on process skills are each evaluated using a 4-point scale in accordance with the capability of the patient. The measured values for motor and process skills are calculated from the evaluation results by interval scale (logits).

\section{Procedure}

All evaluations by CDR, FIM, HADLS, and AMPS were performed within 1 week of hospitalization for each patient.

CDR was evaluated after the first author had a discussion with the attending nurse. FIM was evaluated by the first author based on observations of the patients' behavior in the ward and information on their living conditions from the attending nurse. HADLS was evaluated by the first author based on an interview with live-in relatives or persons familiar with the patients' living conditions immediately before hospitalization. AMPS was also evaluated by the first author.

Data Analysis

ROC Curves for FIM, HADLS, and AMPS

The correlations between CDR and FIM-M, FIM-C, HADLS-ADL, HADLS-IADL, AMPSmotor skills, and AMPS-process skills were calculated; a significant correlation was revealed 
Table 2. AUC, cutoff value, sensitivity, and specificity in the various evaluations

\begin{tabular}{|c|c|c|c|c|c|c|c|}
\hline & \multirow[t]{2}{*}{ AUC } & \multirow[t]{2}{*}{$p$} & \multicolumn{2}{|c|}{$\begin{array}{l}95 \% \text { confidence } \\
\text { interval }\end{array}$} & \multirow[t]{2}{*}{$\begin{array}{l}\text { Cutoff } \\
\text { value }\end{array}$} & \multirow[t]{2}{*}{$\begin{array}{l}\text { Sensitivity, } \\
\%\end{array}$} & \multirow[t]{2}{*}{$\begin{array}{l}\text { Specificity, } \\
\%\end{array}$} \\
\hline & & & $\begin{array}{l}\text { lower } \\
\text { limit }\end{array}$ & $\begin{array}{l}\text { upper } \\
\text { limit }\end{array}$ & & & \\
\hline FIM-M & 0.783 & 0.000 & 0.627 & 0.893 & 62.0 & 72.0 & 68.0 \\
\hline FIM-C & 0.956 & 0.000 & 0.909 & 1.000 & 16.0 & 88.0 & 88.0 \\
\hline HADLS-ADL & 0.846 & 0.000 & 0.749 & 0.943 & 22.40 & 74.0 & 80.0 \\
\hline HADLS-IADL & 0.820 & 0.000 & 0.719 & 0.922 & 35.00 & 74.0 & 84.0 \\
\hline AMPS-motor skills & 0.574 & 0.149 & 0.438 & 0.711 & 1.43 & 74.0 & 48.0 \\
\hline AMPS-process skills & 0.947 & 0.000 & 0.900 & 0.994 & 0.04 & 94.0 & 88.0 \\
\hline
\end{tabular}

AMPS, Assessment of Motor and Process Skills; AUC, area under the curve; FIM-C, Functional Independence Measure Cognition; FIM-M, Functional Independence Measure Motor; HADLS-ADL, Hyogo Activities of Daily Living Scale Activities of Daily Living; HADLS-IADL, Hyogo Activities of Daily Living Scale Instrumental Activities of Daily Living.

for all (rs: -0.235 to -0.855$)$. The ROC curves were prepared for all six evaluation methods that correlated significantly with CDR in order to discriminate CDR2 and CDR3 AD, and AUC values were calculated to investigate the cutoff values, sensitivity, and specificity.

Investigation of Discrimination Precision between CDR2 and CDR3

To determine the combinations of the ADL and IADL evaluation methods (FIM-M, FIM-C, HADLS-ADL, HADLS-IADL, AMPS-motor skills, and AMPS-process skills) that enable a precise discernment between severities of CDR2 and CDR3 AD, discriminant analysis (stepwise method) was performed using discrimination of CDR2 and CDR3 as the dependent variable, and FIM-M, FIM-C, HADLS-ADL, HADLS-IADL, AMPS-motor skills, and AMPS-process skills as the independent variables. Subsequently, ROC curves were constructed to confirm the precision of the evaluations that were selected based on linear discrimination and stepwise method in discriminating CDR2 and CDR3. The significant differences in the calculated AUC were subsequently investigated.

AUC comparisons were performed using EZR version 1.32 (Saitama Medical Center, Jichi Medical University, Saitama, Japan) [11], which is a graphical user interface for the R Foundation for Statistical Computing (R version 2.13.0). SPSS Statistics (Ver. 21.0, IBM Corporation, Armonk, USA) was used for all the other analyses. All $p$ values were two sided and $p$ values less than 0.05 were considered statistically significant.

\section{Results}

ROC Curves of FIM, HADLS, and AMPS

AUC values were estimated from the ROC curves that were constructed for the discrimination of CDR2 and CDR3 AD. With FIM-M, AUC was 0.783 (95\% confidence interval [CI]: $0.627-0.893, p=0.000$ ); at the cutoff value of 62.0 points, sensitivity and specificity were 72.0 and $68.0 \%$, respectively. With FIM-C, AUC was 0.956 (95\% CI: $0.909-1.000, p=0.000$ ); at the cutoff value of 16.0 points, sensitivity and specificity were 88.0 and $88.0 \%$, respectively. With HADLS-ADL, AUC was 0.846 (95\% CI: 0.749-0.943, $p=0.000$ ); at the cutoff value of 22.40 points, sensitivity and specificity were 74.0 and $80.0 \%$, respectively. With HADLS-IADL, AUC 
Fig. 1. Receiver operating characteristic curves of FIM-M, FIM-C, HADLS-ADL, HADLS-IADL, AMPSmotor skills, AMPS-process skills, and discrimination score. FIM-M $(p=0.000)$, FIM-C $(p=0.049)$, HADLS-ADL $(p=0.004)$, HADLSIADL ( $p=0.001)$, and AMPS-motor skills ( $p=0.000)$ were significantly different from the discrimination score. AMPS, Assessment of Motor and Process Skills; FIM-C, Functional Independence Measure Cognition; FIM-M, Functional Independence Measure Motor; HADLS-ADL, Hyogo Activities of Daily Living Scale Activities of Daily Living; HADLS-IADL, Hyogo Activities of Daily Living Scale Instrumental Activities of Daily Living.

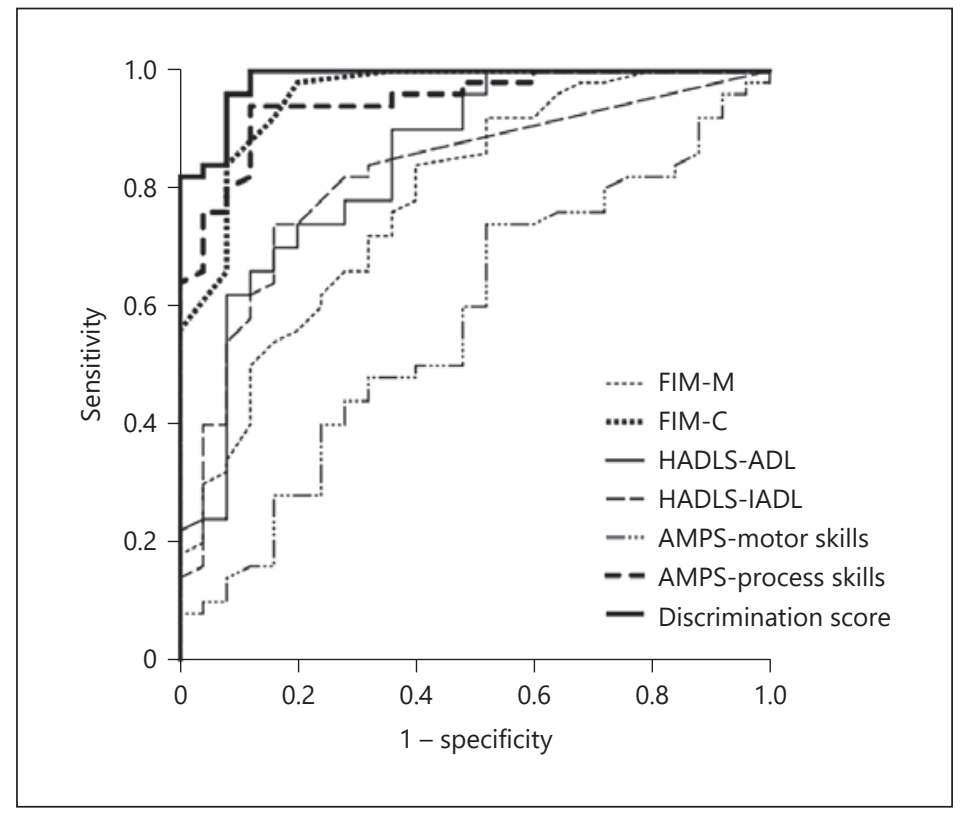

was 0.820 (95\% CI: $0.719-0.922, p=0.000)$; at the cutoff value of 35.00 points, sensitivity and specificity were 74.0 and $84.0 \%$, respectively. With AMPS-motor skills, AUC was 0.574 (95\% CI: $0.438-0.711, p=0.149$ ); at the cutoff value of 1.43 logits, sensitivity and specificity were 74.0 and 48.0\%, respectively. With AMPS-process skills, AUC was 0.947 (95\% CI: 0.900$0.994, p=0.000$ ); at the cutoff value of 0.04 logits, sensitivity and specificity were 94.0 and $88.0 \%$, respectively (Table 2, Fig. 1).

\section{Discrimination Precision for CDR2 and CDR3}

Discriminant Analysis and ROC Curves of the Discrimination Scores

Discriminant analysis was performed with discrimination of CDR2 and CDR3 as the dependent variables, and FIM-M, FIM-C, HADLS-ADL, HADLS-IADL, AMPS-motor skills, and AMPS-process skills as the independent variables. The results revealed that when FIM-C and AMPS-process skills were selected, the linear discriminant was $0.213 \times$ FIM-C $+0.959 \times$ AMPS-process skills - 3.911. Wilk's $\lambda$ (random) was $0.343(p=0.000)$ and the true discrimination rate was $90.7 \%$ (Table 3 ).

When the ROC curve for discriminating CDR2 and CDR3 was constructed using the discriminant scores obtained from the linear discriminant $(0.213 \times$ FIM-C $+0.959 \times$ AMPSprocess skills - 3.911), AUC was 0.985 (95\% CI: 0.963-1.000, $p=0.000)$, and sensitivity and specificity were 96.0 and $92.0 \%$, respectively, at the cutoff value of -0.604 (Fig. 1).

Comparison of AUC Values

Comparison of AUC values of two items revealed that FIM-M $(p=0.002)$, HADLS-ADL ( $p=0.019)$, HADLS-IADL $(p=0.007)$, and AMPS-motor skills $(p=0.000)$ were significantly different from FIM-C (AUC $=0.956)$. In addition, FIM-M $(p=0.004)$, HADLS-ADL $(p=0.029)$, HADLS-IADL ( $p=0.008)$, and AMPS-motor skills $(p=0.000)$ were significantly different from AMPS-process skills (AUC $=0.947$ ).

There was no significant difference between FIM-C (AUC $=0.956)$ and AMPS-process skills (AUC $=0.947, p=0.803$ ) and between the discrimination score (AUC $=0.985$ ) and AMPS-process skills (AUC $=0.947, p=0.119$ ). 
Table 3. Results of the discrimination analysis

\begin{tabular}{llll}
\hline & $\begin{array}{l}\text { Standardized canonical } \\
\text { discriminant function coefficient }\end{array}$ & $\begin{array}{l}\text { Canonical discriminant } \\
\text { function coefficient }\end{array}$ & $p$ value \\
\hline FIM-C & 0.723 & 0.213 & 0.000 \\
AMPS-process skills & 0.486 & 0.959 & 0.000 \\
\hline
\end{tabular}

At Wilks's $\lambda$ of $0.343(p=0.000)$, canonical rate was $90.7 \%$, linear discriminant $(0.213 \times$ FIM-C $+0.959 \times$ AMPS-process skills - 3.911). FIM-C, Functional Independence Measure Cognition; AMPS, Assessment of Motor and Process Skills.

Table 4. Comparison of AUC values in the various evaluations

\begin{tabular}{llll}
\hline & FIM-C & AMPS-process skills & Discrimination score \\
\hline FIM-M & $0.002^{* *}$ & $0.004^{* *}$ & $0.000^{* *}$ \\
FIM-C & - & 0.803 & $0.049^{*}$ \\
HADLS-ADL & $0.019^{*}$ & $0.029^{*}$ & $0.004^{* *}$ \\
HADLS-IADL & $0.007^{*}$ & $0.008^{* *}$ & $0.001^{* *}$ \\
AMPS-motor skills & $0.000^{* *}$ & $0.000^{* *}$ & $0.000^{* *}$ \\
AMPS-process skills & 0.803 & - & 0.119 \\
\hline
\end{tabular}

${ }^{*} p<0.05,{ }^{* *} p<0.01$. AMPS, Assessment of Motor and Process Skills; AUC, area under the curve; FIM-C, Functional Independence Measure Cognition; FIM-M, Functional Independence Measure Motor; HADLS-ADL, Hyogo Activities of Daily Living Scale Activities of Daily Living; HADLS-IADL, Hyogo Activities of Daily Living Scale Instrumental Activities of Daily Living.

FIM-M ( $p=0.000)$, FIM-C ( $p=0.049)$, HADLS-ADL ( $p=0.004)$, HADLS-IADL $(p=0.001)$, and AMPS-motor skills $(p=0.000)$ were significantly different from the discrimination score (AUC $=0.992)$ (Table 4, Fig. 1).

\section{Discussion}

\section{ROC Curves of FIM, HADLS, and AMPS}

To determine which evaluation will enable the most precise and robust discrimination between CDR2 and CDR3 AD, we plotted ROC curves of these evaluations to calculate AUC values and investigate the related cutoff values, sensitivity, and specificity.

We found that AUC values for process skills determined by FIM-C and AMPS were 0.956 and 0.947 , respectively, indicating values of $\geq 0.9$ only for these two evaluations. Swets [12] previously reported prediction rates obtained using AUC; the accuracy was judged as high, moderate, and low for rates $\geq 0.9,0.7-0.9$, and $0.5-0.7$, respectively. Based on these findings, our AUC results indicated that only FIM-C and AMPS-process skills had a high accuracy in effectively discriminating between CDR2 and CDR3 AD. In other words, it became evident that this differentiation can be accurately determined using appropriate methods. To our knowledge, this is the first study to report such findings.

Based on the results, FIM-C, which is strongly affected by cognitive functions, was strongly correlated with MMSE ( $r s=0.843, p<0.01)$ and CDR ( $r s=-0.909, p<0.01)$, suggesting severe dementia. CDR evaluates memory, orientation, judgment and problem solving, community affairs, home and hobbies, and personal care, each on a 5-point scale and based on clinical 
information and patients' observed behavior. Severity of dementia is comprehensively judged and strongly reflects diminished cognitive function. These findings suggested that FIM-C enables accurate determination of differences in severity between CDR2 and CDR3 AD.

Moreover, communication ability, i.e., understanding based on hearing, sight, and verbal and nonverbal expressions, is included in the FIM-C evaluation. Decreased communication ability in patients with AD manifests consistently as AD advances [13], and the decrease becomes profound from CDR2 onwards [4]. However, in a clinical setting, even among CDR2 and CDR3 AD patients, many can still effectively express their intentions, desires, and feelings and some can understand the nonverbal expressions of the occupational therapist. Indeed, in clinical settings, the range of individual differences in communication abilities across CDR2 and CDR3 AD patients can be quite remarkable.

Although communication ability is not among the CDR evaluation items, it was involved in the results of evaluation items such as judgment power, problem solving, social adjustment, and understanding family situations. Therefore, high AUC value of FIM-C for discriminating between CDR2 and CDR3 AD was likely a major contributing factor in enabling the evaluation of communication ability by FIM-C.

\section{Investigating the Precision of Discriminating between CDR2 and CDR3}

Discriminant analysis was performed with CDR2 and CDR3 as dependent variables and FIM-M, FIM-C, HADLS-ADL, HADLS-IADL, AMPS-motor skills, and AMPS-process skills as independent variables. Based on the results, FIM-C and AMPS-process skills were selected as evaluations. Moreover, comparison of the AUC values of both methods revealed that FIM-C differed significantly from FIM-M, HADLS-ADL, HADLS-IADL, and AMPS-motor skills. In addition, AMPS-process skills differed significantly from FIM-M, HADLS-ADL, HADLSIADL, and AMPS-motor skills. This demonstrated that FIM-C and AMPS-process skills are highly precise ADL/IADL evaluations for discriminating between CDR2 and CDR3 AD.

Comparison of AUC values also revealed significant differences in the discrimination score (FIM-C and AMPS-process skills combined) among FIM-M, FIM-C, HADLS-ADL, HADLSIADL, and AMPS-motor skills. This confirmed that FIM-C and AMPS-process skills combination is a highly precise approach for discriminating between CDR2 and CDR3 AD. In particular, as there were significant differences between the discrimination score and FIM-C, the FIM-C plus AMPS-process skills combination, compared with FIM-C alone, could increase the discrimination score and improve the possibility of better discrimination between the severity of CDR2 and CDR3 AD. These results indicate that even with a marked decrease in cognitive function, as evidenced by FIM-C results, the evaluation of ADL/IADL by AMPSprocess skills enables the identification of various spared abilities of the patients. Thus, evaluation of patients with CDR2 and CDR3 AD using FIM-C and AMPS-process skills combination provides useful information for establishing occupational therapy programs that maximize the use of intact skills in daily living activities in AD patients.

AMPS-process skills enables the identification of current capabilities that may become problems in daily living, as well as intact skills that are strongly influenced by cognitive capabilities. However, AMPS-process skills do not address communication ability, which is markedly decreased in patients with moderate to advanced AD. In contrast, FIM-C assesses both cognitive function and communication ability. Thus, the combination of these two methods enables more accurate discrimination between CDR2 and CDR3 AD.

\section{Disclosure Statement}

The authors declare no conflict of interest. 


\section{References}

1 Nakamura Y, Sinkoushita AD. Heno chiryou apurouchi no arikata wo kangaeru - koudo AD no byotai wo saikousuru - [in Japanese]. Japanese Journal of Geriatric Psychiatry. 2008 Jan;19:104-14.

2 Otoyama W, Niina R, Homma A, Sanada J, Takahashi M, Kamimura N, et al. Inter-rater reliability of the Japanese version of Clinical Dementia Rating (CDR) [in Japanese]. Japanese Journal of Geriatric Psychiatry. 2000;11(5): 521-7.

3 Tröster AI. Neuropsychological characteristics of dementia with Lewy bodies and Parkinson's disease with dementia: differentiation, early detection, and implications for "mild cognitive impairment" and biomarkers. Neuropsychol Rev. 2008 Mar;18(1):103-19.

4 Choe JY, Youn JC, Park JH, Park IS, Jeong JW, Lee WH, et al. The Severe Cognitive Impairment Rating Scale-an instrument for the assessment of cognition in moderate to severe dementia patients. Dement Geriatr Cogn Disord. 2008;25(4):321-8.

5 Ottenbacher KJ, Hsu Y, Granger CV, Fiedler RC. The reliability of the functional independence measure: a quantitative review. Arch Phys Med Rehabil. 1996 Dec;77(12):1226-32.

6 Hirono N, Mori E, Yamashita H, Tokimasa S, Yamadori S. A novel scoring system of activities of daily living for patients with Alzheimer's disease: Hyogo Activities of Daily Living Scale (HADLS) [in Japanese]. Jpn J Neuropsychol. 1997;13(4):260-9.

7 Fisher AG, Jones KB. Evaluation of motor and process skills. Volume 1. 7th ed., Revised. Fort Collins (Colorado): Three Star Press; 2012.

8 Fisher AG, Jones KB. Evaluation of motor and process skills. Volume 2. 7th ed., Revised. Fort Collins (Colorado): Three Star Press; 2012.

9 Tanaka H, Uematsu M, Koshiro R, Nagata Y, Fukuhara K, Naito Y, et al. Association of cognitive function, activities of daily living and behavioral and psychological symptoms of dementia in dementia patients - Focusing on severe dementia patients - [in Japanese]. Japanese Journal of Geriatric Psychiatry. 2014;25(3):316-23.

10 Sugiyama M, Ijuin M, Sakuma N, Miyamae F, Ito K, Ura T, et al. Reliability and validity of the Five Cognitive Test in the context of detecting older people with mild cognitive impairment living in the community [in Japanese]. Japanese Journal of Geriatric Psychiatry. 2015;26(2):183-95.

11 Kanda Y. Investigation of the freely available easy-to-use software 'EZR' for medical statistics. Bone Marrow Transplant. 2013 Mar;48(3):452-8.

12 Swets JA. Measuring the accuracy of diagnostic systems. Science. 1988 Jun;240(4857):1285-93.

13 Ripich DN, Carpenter BD, Ziol EW. Conversational cohesion patterns in men and women with Alzheimer's disease: a longitudinal study. Int J Lang Commun Disord. 2000 Jan-Mar;35(1):49-64. 